\title{
Is the Planck's Length the Shortest Meaningful Length in Space-time?
}

\author{
Branko M. Novakovic
}

\begin{abstract}
The Planck's length $L_{p}$, is believed to be the shortest meaningful length as the limiting distance below which the regular notions of space and length surcease to exist. Any attempt to investigate the possible existence of smaller distances, by using higher energy collisions, would result in black hole production. This is the consequence of the vacuum solution of the Einstein's field equations that predicts the singularities and the related black holes in the extremely strong field. Recently, it has been developed a new Relativistic Alpha Field Theory (RAFT) that extends the application of General Relativity Theory (GRT) to the extremely strong fields at the Planck's scale. One of the predictions of RAF theory is: there exists a minimal gravitational radius at $r=\left(G M / 2 c^{2}\right)$ that prevents singularity at $r=0$, i.e. the nature protects itself. If RAF theory is correct then one can find out the following consequences of the existence of the minimal radius in a gravitational field: a) each mass has its own minimal radius, b) the minimal radius of the Planck's mass is equal to half of the Planck's length, c) the smallest minimal radius in a gravitational field belongs to the particle with the smallest mass and d) the Planck's mass is not the smallest mass in the spacetime and therefore the Planck's length is not the shortest meaningful length. Thus, it has been theoretically confirmed that the physical significance of the Planck's length is the minimal length (diameter) of the Planck's mass.
\end{abstract}

Index Terms- Meaningful minimal length, Planck's length, Relativistic Alpha Field Theory (RAFT), Extremely strong gravitational fields

\section{INTRODUCTION}

The Planck's length, $L_{p}$, [1-6] and Planck's mass, $M_{p}$, [7-9] are the base units in the system of natural units known as Planck's units that have been developed by physicist Max Planck. It is believed that the Planck's length is the shortest meaningful length as the limiting distance below which the regular notions of space and length surcease to exist. There is currently no proven physical significance of the Planck's length. Meanwhile, it is theoretically considered to be the quantization of space in the quantum gravity theory [4]. In some forms of quantum gravity, the Planck's length is the length scale at which the structure of spacetime becomes dominated by quantum effects, and it is impossible to determine the difference between two locations less than one Planck' length apart. Any attempt to investigate the possible existence of smaller distances, by using higher energy collisions, would result in black hole production [3]. This is the consequence of the vacuum solution of the Einstein's field equations that predicts the singularities in the extremely strong field and the existence of the related black holes. Therefore, General Relativity Theory (GRT) [10-15] with

Branko Novakovic, FSB - University of Zagreb, Luciceva 5, P.O.B. 509, 10000 Zagreb, Croatia. vacuum solution of the Einstein's field equations cannot be applied to the extremely strong field at Planck's scale.

Recently, it has been developed a new Relativistic Alpha Field Theory (RAFT) [16-21,25] that extends the application of GRT to the extremely strong fields including of the Planck's scale [22-24]. This is the consequence of the following predictions of RAF theory [16-19]: a) no a singularity at the Schwarzschild radius, b) there exists a minimal radius at $r_{m}=\left(G M / 2 c^{2}\right)$ that prevents singularity at $r$ $=0$, i.e. the nature protects itself, and c) the gravitational force becomes positive (repulsive) if $\left(G M / r c^{2}\right)>1$, that could be a source of a dark energy.

Here we started with the investigation of the minimal gravitational radius $r_{p m}$ of the Planck's mass $M_{p}$. In order to apply RAF theory, we assumed that the Planck's mass $M_{p}$ is the spherically symmetric non-rotating body. Further we used the relation for the minimal radius in a gravitational field [16-18] and obtained that the Planck's length $L_{p}$ is the diameter of the Planck's mass. Consequently, the minimal gravitational radius $r_{p m}$ of the Planck's mass is equal to half of the Planck's length: $r_{p m}=\left(G M_{p} / 2 c^{2}\right)=L_{p} / 2$. On that way, it has been theoretically confirmed that the physical significance of the Planck's length is the minimal length (diameter) of the Planck's mass.

Further we wanted to know does the Planck's length is the smallest length in a spacetime? The next investigation result tells us that the smallest minimal radius $r_{m}$ (the smallest diameter length) in a gravitational field belongs to the particle with the smallest mass $M_{m}$. Since the Planck's mass $M_{p}$, is not the smallest mass in the spacetime, one can conclude that the Planck's length $L_{p}$ is not the shortest meaningful length as the limiting distance below which any regular smaller distance in a gravitational field cannot exist. Since each i-th mass $M_{i}$ has its own minimal i-th gravitational radius $r_{m i}$, the radial mass density $\rho_{r}=M / r$ of each i-th mass at i-th minimal gravitational radius is constant and equal to $\rho_{r}=2 c^{2} / G$. This radial density is the same for all $\left(M_{i}, r_{m i}\right)$ pairs of masses and minimal gravitational radiuses. At the same time, this radial density is the maximal one in the gravitational field, $\rho_{\text {rmax }}=2 c^{2} / G$.

It is of the grate interest to investigate the radial acceleration at the minimal gravitational radius, $\ddot{r}_{m}$. The results of that investigation showed that the radial acceleration at the minimal gravitational radius is given by the relation $\ddot{r}_{m}=C / M$, where $M$ is mass and $C$ is constant. This constant can be described by three relations: $4 c^{4} / G$, or $\rho_{\text {rmax }}^{2} G$, or $4 c^{4} M_{p} / L_{p}$. In the case that mass is equal to the Planck's mass, $M=M_{p}$, the radial acceleration at the minimal Planck's radius $r_{p m}=L_{p} / 2$ is given by the relation $\ddot{r}_{m}=4 c^{4} / L_{p}$. From the relation of the radial acceleration at the minimal gravitational radius we can see that the maximal acceleration belongs to the smallest mass. It is very important to point out 
that the radial acceleration at the minimal gravitational radius is positive (repulsive) one and could be the source of the dark energy. More details about that have been presented in [18, 19].

Furthermore, the existence of the minimal gravitational radius, predicted by RAF theory, has been employed for derivation of the new quantum gravity model [25]. The existence of a non-singular rotating black hole, presented in [26], could also be the consequence of the existence of the minimal gravitational radius. In [27-32] different problems have been considered by applying Planck's parameters $\left(c, \hbar, G, L_{p}, M_{p}\right)$. The mentioned problems can also be solved by using maximal radial density in the gravitational field, $\rho_{\text {rmax }}=2 c^{2} / G$, that has been defined in this article.

\section{DeRVATION OF Minimal Radius of PlanCK’s Mass}

An alpha field is a potential field that can be described by two field parameters $\alpha$ and $\alpha^{\prime}$. To this category belong, among the others, electrical and gravitational fields. Field parameters $\alpha$ and $\alpha^{\prime}$ are described as the scalar dimensionless (unitless) functions of the potential energy $U$ of a particle in an alpha field [16]. In order to determine minimal gravitational radius of the Planck's mass, one can start with the general line element $\mathrm{ds}^{2}$ derived in Relativistic Alpha Field Theory (RAFT) [16]

$$
\begin{aligned}
d s^{2}= & -\alpha \alpha^{\prime} c^{2} d t^{2}-\kappa\left(\alpha-\alpha^{\prime}\right)_{x} c d t d x-\kappa\left(\alpha-\alpha^{\prime}\right)_{y} c d t d y \\
& -\kappa\left(\alpha-\alpha^{\prime}\right)_{z} c d t d z+d x^{2}+d y^{2}+d z^{2}
\end{aligned}
$$

Here $\kappa$ is a constant. Following the well-known procedure [10-15], this line element can be transformed into the spherical polar coordinates in the nondiagonal form

$$
\begin{aligned}
d s^{2}= & -\alpha \alpha^{\prime} c^{2} d t^{2}-\kappa\left(\alpha-\alpha^{\prime}\right) c d t d r+d r^{2}+r^{2} d \theta^{2} \\
& +r^{2} \sin ^{2} \theta d \phi^{2}
\end{aligned}
$$

The line element (2) belongs to the well-known form of the Riemann's type line element [10-15]

$$
\begin{aligned}
d s^{2}= & g_{00}\left(d x^{0}\right)^{2}+2 g_{01} d x^{0} d x^{1}+g_{11}\left(d x^{1}\right)^{2} \\
& +g_{22}\left(d x^{2}\right)^{2}+g_{33}\left(d x^{3}\right)^{2} .
\end{aligned}
$$

Comparing the equations (2) and (3) we obtain the coordinates and components of the covariant metric tensor, valid for the line element (2):

$$
\begin{gathered}
d x^{0}=c d t, \quad d x^{1}=d r, \quad d x^{2}=d \theta, \quad d x^{3}=d \phi, \\
g_{00}=-\alpha \alpha^{\prime}, \quad g_{01}=g_{10}=\frac{-\kappa\left(\alpha-\alpha^{\prime}\right)}{2}, \\
g_{11}=1, \quad g_{22}=r^{2}, \quad g_{33}=r^{2} \sin ^{2} \theta .
\end{gathered}
$$

Starting with the line element (2) we employ, for the convenient, the following substitutions:

$$
v=\alpha \alpha^{\prime}, \quad \lambda=\kappa\left(\alpha^{\prime}-\alpha\right) / 2 .
$$

In that case the nondiagonal line element (2) is transformed into the new relation $d s^{2}=-v c^{2} d^{2}+2 \lambda c d t d r+d r^{2}+r^{2} d \theta^{2}+r^{2} \sin ^{2} \theta d \phi^{2}$.

Using the coordinate system (4), the related covariant metric tensor $g_{\mu \eta}$ of the line element (6) is presented by the matrix form [16]:

$$
\left[g_{\mu \eta}\right]=\left[\begin{array}{cccc}
-v & \lambda & 0 & 0 \\
\lambda & 1 & 0 & 0 \\
0 & 0 & r^{2} & 0 \\
0 & 0 & 0 & r^{2} \sin ^{2} \theta
\end{array}\right] .
$$

This tensor is symmetric and has six non-zero elements as we expected that should be.

For calculation of the field parameters $\alpha$ and $\alpha^{\prime}$ we have to use two equations. The first one is the Einstein's field equations [10-15] without a cosmological constant $(\Lambda=0)$

$$
R_{\mu \eta}-\frac{1}{2} g_{\mu \eta} R=k T_{\mu \eta}, k=\frac{8 \pi G}{c^{4}}, \mu, \eta=0,1,2,3 .
$$

Here $R_{\mu \eta}$ is the Ricci tensor, $g_{\mu \eta}$ is the metric tensor, $R$ is the Ricci scalar and $T_{\mu \eta}$ is the gravitational energy-momentum tensor. The second equation is the covariant energy equation $E_{c}$, valid for an alpha field [20]:

$$
\begin{aligned}
& E_{c}=H m_{0} \alpha \alpha^{\prime} c^{2}+\frac{H m_{0} \kappa\left(\alpha-\alpha^{\prime}\right) c V}{2}, \\
& H=\left(\alpha \alpha^{\prime}-\frac{V^{2}}{c^{2}}+\frac{\kappa\left(\alpha-\alpha^{\prime}\right) c V}{c^{2}}\right)^{-1 / 2} .
\end{aligned}
$$

Here $m_{0}$ is a particle rest mass, $V$ is a relative velocity in vacuum, $\kappa= \pm 1$ and $H$ is the transformation factor in an alpha field which, for the case without any potential field $(\alpha=$ $\left.\alpha^{\prime}=1\right)$, is reduced to the Lorentz - Einstein transformation factor $\gamma$, valid in the Special Relativity. From the equation (9) we can see that the covariant energy equation $E_{c}$ is in the linear form. The related nonlinear equation of $E_{c}$ can be obtained by applying of the square operation to the relation (9) (see [20]):

$$
\frac{E_{c}^{2}}{c^{2}}-P^{2}=m_{0} \alpha \alpha^{\prime} c^{2}, \quad P=H m_{0} V .
$$

This is the nonlinear energy - momentum equation of a particle moving in an alpha field with a relativistic momentum $P$. This new nonlinear relation is form invariant and consistent to the related equation in the Special Relativity.

Applying relations (5-9) we obtain the solutions of the field parameters $v$ and $\lambda$ in a gravitational static field [16]:

$$
\begin{aligned}
& v=\left(1-\frac{G M}{r c^{2}}\right)^{2}=\left(1-\frac{2 G M}{r c^{2}}+\left(\frac{G M}{r c^{2}}\right)^{2}\right), \\
& \lambda=\sqrt{\frac{2 G M}{r c^{2}}-\left(\frac{G M}{r c^{2}}\right)^{2}} \rightarrow v=1-\lambda^{2} .
\end{aligned}
$$

The related solution of the components of the energy-momentum tensor in the gravitational spherically symmetric static field is given by relation [17] 


$$
\begin{aligned}
T_{\mu \eta} & =\left[T_{00}, T_{01}, T_{10}, T_{11}, T_{22}, T_{33}\right] \\
& =\left[\nu,-\lambda,-\lambda,-1, r^{2}, r^{2} \sin ^{2} \theta\right] \frac{(G M)^{2}}{8 \pi G r^{4}} .
\end{aligned}
$$

Thus, applying the relations ( 6 - 11), we obtain solution of the line element for the gravitational spherically symmetric static field

$$
\begin{aligned}
d s^{2}= & -\left(1-\frac{G M}{r c^{2}}\right)^{2} c^{2} d t^{2}+2 \sqrt{\frac{2 G M}{r c^{2}}-\left(\frac{G M}{r c^{2}}\right)^{2}} c d t d r \\
& +d r^{2}+r^{2} d \theta^{2}+r^{2} \sin ^{2} \theta d \phi^{2} .
\end{aligned}
$$

Remarks 1 . The field parameters (11) satisfy the Einstein's field equations (8) with a cosmological constant $\Lambda=0$ and the covariant energy equation $E_{c}(9)$. In the case of a strong static gravitational field [22-24], the quadratic term $\left(G M / r c^{2}\right)^{2}$ generates the related energy-momentum tensor $T_{\mu \eta}(12)$. For that case, we do not need to add by hand the related energy-momentum tensor $T_{\mu \eta}$ on the right side of the Einstein's field equations (8). In the case of a relatively weak static gravitational field, like in our solar system, the quadratic term $\left(G M / r c^{2}\right)^{2}$ is too small and can be neglected. For that case, the field parameters (11) satisfy the Einstein's field equations in a vacuum $\left(T_{\mu \eta}=0, \Lambda=0\right)$, and line element (13) is converted into the related vacuum solution

$$
\begin{aligned}
d s^{2}= & -\left(1-\frac{2 G M}{r c^{2}}\right) c^{2} d t^{2}+2 \sqrt{\frac{2 G M}{r c^{2}}} c d t d r \\
& +d r^{2}+r^{2} d \theta^{2}+r^{2} \sin ^{2} \theta d \phi^{2} .
\end{aligned}
$$

Following the solution of the line element (13) one can conclude that RAF theory extends GR to the extremely strong gravitational field including Planck's scale.

Proposition 1. Considering the solution of the line element in the extremely strong gravitational field (13) we can conclude that the minimal radius of the Planck's mass $M_{p}$, denoted by $r_{p m}$, is equal to half of the Planck's length $L_{p}$ :

$$
r_{p m}=\frac{L_{p}}{2}, \rightarrow L_{p}=2 r_{p m} .
$$

Proof of the Proposition 1. In order to prove of the proposition 1 we can start with the line element (13). This line element can be rewritten into the following form

$$
\begin{aligned}
d s^{2}= & -\left(1-\frac{G M}{r c^{2}}\right)^{2} c^{2} d t^{2}+2 \sqrt{\frac{2 G M}{r c^{2}}\left(1-\frac{G M}{2 r c^{2}}\right)} c d t d r \\
& +d r^{2}+r^{2} d \theta^{2}+r^{2} \sin ^{2} \theta d \phi^{2} .
\end{aligned}
$$

It is easy to prove that the line element (16) is regular if the following condition is satisfied:

$$
\left(1-\frac{G M}{2 r c^{2}}\right) \geq 0 \rightarrow \frac{G M}{2 r c^{2}} \leq 1 \rightarrow \frac{G M}{2 c^{2}} \leq r<\infty .
$$

Thus, the region of the regularity of the line element (16) is determined by the last relation in (17). From the previous relation we can conclude that there exists the minimal gravitational radius $r_{m}$ which still preserves regularity of the line element (16)

$$
r_{m}=\frac{G M}{2 c^{2}} .
$$

Thus, including of the minimal gravitational radius $r_{m}(18)$ into the line element (16) we obtain regular line element in the form

$$
d s^{2}=-c^{2} d t^{2}+d r^{2}+r^{2} d \theta^{2}+r^{2} \sin ^{2} \theta d \phi^{2} .
$$

For the radiuses less than minimal gravitational radius $r_{m}$ the line element (16) becomes imaginary item. Compare the minimal gravitational radius $r_{m}$ with the Schwarzschild radius $r_{s}$ one can see that the minimal gravitational radius is four time less than Schwarzschild radius

$$
r_{s}=\frac{2 G M}{c^{2}}=4 r_{m}=4 \frac{G M}{2 c^{2}} .
$$

Now, applying of the Planck's mass $M_{p}$ to the relation (18) we obtain minimal gravitational radius of the Planck's mass

$$
r_{p m}=\frac{G M_{p}}{2 c^{2}} .
$$

As it is well-known, the Planck's mass $M_{p}$ [7-9] and the Planck's length $L_{p}[1-6]$ are defined from three fundamental physical constants: the speed of light in vacuum $c$, the reduced Planck's constant $\hbar$ and the gravitational constant $G$ :

$$
M_{p}=\sqrt{\frac{\hbar c}{G}}, \quad L_{p}=\sqrt{\frac{\hbar G}{c^{3}}} .
$$

Assuming that the Planck's mass $M_{p}$ has spherically symmetric form (i.e. $L_{p}$ is diameter of the Planck's mass) and following the relations (21) and (22), we can calculate the following equality:

$$
\begin{aligned}
\frac{M_{p}}{2 r_{p m}} & =\frac{c^{2}}{G}, \frac{M_{p}}{L_{p}}=\frac{\sqrt{\hbar c / G}}{\sqrt{\hbar G / c^{3}}}=\frac{c^{2}}{G}=\text { const. } \\
& \rightarrow 2 r_{p m}=L_{p} \rightarrow r_{p m}=\frac{L_{p}}{2} .
\end{aligned}
$$

By the last relation in (23), the proof of the proposition 1 (that the minimal gravitational radius of the Planck's mass $M_{p}$, denoted by $r_{p m}$, is equal to half of the Planck's length $L_{p}$ ) is finished.

\section{Is THE PlanCK'S LeNGTH THE SHORTEST MEANINGFUL LENGTH IN SPACETIME?}

The Planck's length, $L_{p}$, [1-6] is believed to be the shortest meaningful length as the limiting distance below which any regular smaller distance in the spacetime cannot exist. There is currently no proven physical significance of the Planck's length. Any attempt to investigate the possible existence of smaller distances, by using higher energy collisions, would result in black hole production [3]. This is the consequence of the vacuum solution of the Einstein's field equations that 
predicts the singularities in the extremely strong field and the production of the related black holes. Recently, it has been developed a new Relativistic Alpha Field Theory (RAFT) that extends the application of General Relativity Theory (GRT) to the extremely strong fields at the Planck's scale [16-19]. This is the consequence of the following predictions of RAF theory:

a) no a singularity at the Schwarzschild radius,

b) there exists a minimal radius at $r=\left(G M / 2 c^{2}\right)$ that prevents singularity at $r=0$, i.e. the nature protects itself, and

c) the gravitational force becomes positive (repulsive) if $\left(G M / r c^{2}\right)>1$, that could be a source of a dark energy.

Here we investigate the possibility that the gravitational minimal radius $r_{p m}$ of the Planck's mass $M_{p}$ is the regular smallest minimal radius in a gravitational field at all.

Proposition 2. Considering the relations (18) and (21) we can conclude that the smallest minimal radius $r_{m}$ (or the shortest meaningful length $L_{m}=2 r_{m}$ ) in a gravitational field belongs to the particle with the smallest mass $M_{m}$. Since the Planck's mass $M_{p}$, is not the smallest mass in the spacetime, one can conclude that the Planck's length $L_{p}$ is not the shortest meaningful length as the limiting distance below which any regular smaller distance in a gravitational field does not exist:

$$
\begin{aligned}
M_{m}<M_{p}, & \rightarrow r_{m}=\frac{L_{m}}{2}<r_{p m}=\frac{L_{p}}{2}, \\
& \rightarrow L_{m}<L_{p} .
\end{aligned}
$$

Proof of the Proposition 2. In order to prove of the proposition 2 we can start with the general relation for the minimal radius (18)

$$
r_{m}=\frac{G M}{2 c^{2}}=\frac{G}{2 c^{2}} M=K_{p} M .
$$

From the equation (25) we can see that the minimal gravitational radius is proportional to the particle mass, where the constant of proportionality $K_{p}=G / 2 c^{2}$. This means that the all masses less than Planck's mass $M_{p}$ have the minimal gravitational radiuses $r_{m}$ less than the minimal gravitational radius of the Planck's mass $r_{p m}$. Since the Planck's length is equal to $L_{p}=2 r_{p m}$, one can conclude that the Planck's length $L_{p}$ is not the shortest meaningful length as the limiting distance below which any regular smaller distance in a gravitational field does not exist.

For an example we can use mass of the proton $M$. This mass is smaller than the Planck's mass $M_{p}$. Therefore, the minimal gravitational radius of the proton $r_{m}$ is less than minimal gravitational radius of the Planck's mass $r_{p m}$ :

$$
\begin{gathered}
M=1.672621898 \cdot 10^{-27} \mathrm{~kg}, \quad M_{p}=2.176470 \cdot 10^{-8} \mathrm{~kg}, \\
r_{m}=\frac{G M}{2 c^{2}}, \quad r_{p m}=\frac{G M_{p}}{2 c^{2}}, \quad M<M_{p} \rightarrow \\
r_{m}<r_{p m}, \quad \rightarrow \quad 2 r_{m}=L_{m}<2 r_{p m}=L_{p} \rightarrow L_{m}<L_{p} .
\end{gathered}
$$

Consequently, the Planck's length $L_{p}$ is not the shortest meaningful length in a gravitational field at all. By the last relation in (26), the proof of the proposition 2 (that the
Planck's length $L_{p}$ is not the shortest meaningful length in a gravitational field at all) is finished.

For an illustration, here are calculated and presented minimal gravitational radiuses for the proton mass $M$, Planck's mass $M_{p}$, Earth mass $M_{e}$, our Sun mass $M_{s}$ and visible and total masses of the Universe $M_{v}$ and $M_{t}$, respectively:

$$
\begin{gathered}
M=1.672621898 \cdot 10^{-27} \mathrm{~kg} \rightarrow r_{m}=0.6210 \cdot 10^{-54} \mathrm{~m}, \\
M_{p}=2.176470 \cdot 10^{-8} \mathrm{~kg} \rightarrow r_{p m}=0.8081145 \cdot 10^{-35} \mathrm{~m}, \\
M_{e}=5.98 \cdot 10^{24} \mathrm{~kg} \rightarrow r_{e m}=2.2203 \cdot 10^{-3} \mathrm{~m}, \\
M_{s}=1.989 \cdot 10^{30} \mathrm{~kg} \rightarrow r_{s m}=0.7385 \cdot 10^{3} \mathrm{~m}, \\
M_{v}=6 \cdot 10^{51} \mathrm{~kg} \rightarrow r_{v m}=2.2278 \cdot 10^{24} \mathrm{~m}, \\
M_{t}=6 \cdot 10^{52} \mathrm{~kg} \rightarrow r_{t m}=2.2278 \cdot 10^{25} \mathrm{~m} .
\end{gathered}
$$

From (27) we can see that the minimal gravitational radiuses of the both universe masses are quite big.

\section{IS THE MAXIMAL RADIAL DENSITY $\rho_{r \text { max }}=M / r_{\text {min }}$} THE SAME FOR ALL MASSES?

It is very important to consider radial density $\rho_{r_{m_{i}}}=M_{i} / r_{m_{i}}$ at the each $i$-th minimal gravitational radiuses of the related $i$-th mass. Since the minimal gravitational radius is the smallest radius for the related mass, one can anticipate that the radial density at the minimal gravitational radius is the maximal one. But which of them is the maximal maximum?

Proposition 3. From the equation of the minimal gravitational radius (18) one can see that each $i$-th mass $M_{i}$ has its own minimal $i$-th gravitational radius $r_{m i}$. But, the radial density of the each $i$-th mass at the $i$-th minimal gravitational radius is constant and same for all pairs of masses and minimal gravitational radiuses $\left(M_{i}, r_{m_{i}}\right)$. At the same time, this radial density is the maximal one in a gravitational field

$$
\rho_{r_{m_{i}}}=\frac{M_{i}}{r_{m_{i}}}=\frac{2 c^{2}}{G}=\rho_{r_{\max }}=\text { const., } \quad i=1,2, \ldots . .
$$

Proof of the Proposition 3. In order to prove of the proposition 3, one can start with the equation for the minimal gravitational radius (18)

$$
r_{m}=\frac{G M}{2 c^{2}}, \quad \rho_{r_{m}}=\frac{M}{r_{m}}=\frac{2 c^{2}}{G}=\text { const } .
$$

Since each mass has its own minimal radius, the general relation of (29) has the following form:

$$
r_{m_{i}}=\frac{G M_{i}}{2 c^{2}}, \rho_{r_{m_{i}}}=\frac{M_{i}}{r_{m_{i}}}=\frac{2 c^{2}}{G}=\text { const., } i=1,2, . .
$$

The minimal gravitational radius is changing by the mass change. But, the radial density $\rho_{r}$ is the ratio of the mass and radius. At the minimal gravitational radius this ratio is constant and the same for all masses. Since the minimal 
gravitational radius is the smallest one and mass is constant, the radial density at the minimal gravitational radius is the maximal one

$$
\rho_{r_{\max }}=\frac{M_{i}}{r_{m_{i}}}=\frac{2 c^{2}}{G}=\text { const. }, \quad i=1,2, \ldots . .
$$

By the relation (31), the proof of the proposition 3 (that the radial density of the each $i$-th mass at the $i$-th minimal gravitational radius is constant and maximal and the same for the all $\left(M_{i}, r_{m_{i}}\right)$ pairs $)$ is finished.

Finally, one can conclude that the proton mass $M$, Planck's mass $M_{p}$, Earth mass $M_{e}$, our Sun mass $M_{s}$, visible and total masses of the Universe $M_{v}$ and $M_{t}$, as well as the all other masses in the universe have the same maximal radial density

$$
\rho_{r_{\max }}=\frac{2 c^{2}}{G}=2.6932 \cdot 10^{27} \mathrm{~kg} / \mathrm{m} .
$$

Thus, if RAF theory is correct, then the maximal radial density for any mass in gravitational field is given by the relation (32).

\section{RADIAL ACCELERATION AND FORCE AT THE MINIMAL GRAVITATIONAL RADIUS}

Following RAF theory, the geodesic equations for the coordinates $x, y$ and $z$ are described by the relations [18]:

$$
\begin{gathered}
\ddot{x}+\dot{\lambda}_{x} c-\lambda_{x} \lambda^{\prime}{ }_{x} c^{2}=0, \quad \ddot{y}+\dot{\lambda}_{y} c-\lambda y \lambda^{\prime} y c^{2}=0, \\
\ddot{z}+\dot{\lambda}_{z} c-\lambda_{z} \lambda^{\prime}{ }_{z} c^{2}=0, \quad \lambda_{x}=-\kappa\left(\alpha-\alpha^{\prime}\right)_{x} / 2, \\
\lambda_{y}=-\kappa\left(\alpha-\alpha^{\prime}\right)_{y} / 2, \quad \lambda_{z}=-\kappa\left(\alpha-\alpha^{\prime}\right)_{z} / 2, \\
\frac{\partial \lambda x}{\partial c t}=\frac{\dot{\lambda}_{x}}{c}, \quad \frac{\partial \lambda_{y}}{\partial c t}=\frac{\dot{\lambda}_{y}}{c}, \quad \frac{\partial \lambda_{z}}{\partial c t}=\frac{\dot{\lambda}_{z}}{c}, \frac{\partial \lambda_{x}}{\partial x}=\lambda^{\prime} x, \\
\frac{\partial \lambda_{y}}{\partial y}=\lambda^{\prime}{ }_{y}, \frac{\partial \lambda_{z}}{\partial z}=\lambda_{z}^{\prime}, \quad \kappa= \pm 1 .
\end{gathered}
$$

Here $\alpha$ and $\alpha^{\prime}$ are field parameters that have been determined in [16]. For the time-invariant, or very slowly changeable alpha field, the parameters $\dot{\lambda}_{x}=\dot{\lambda}_{y}=\dot{\lambda}_{z}=0$, and the relations (33) are reduced into the simplest forms:

$$
\begin{gathered}
\ddot{x}-\lambda_{x} \lambda^{\prime}{ }_{x} c^{2}=0, \quad \ddot{y}-\lambda_{y} \lambda^{\prime}{ }_{y} c^{2}=0, \\
\ddot{z}-\lambda_{z} \lambda^{\prime}{ }_{z} c^{2}=0 .
\end{gathered}
$$

For the radial coordinate $r$, the geodesic equations (34) are transformed into the following form

$$
\begin{gathered}
\ddot{r}-\lambda_{r} \lambda_{r}^{\prime} c^{2}=0, \quad \ddot{r}=\lambda_{r} \lambda_{r}^{\prime} c^{2}, \\
\lambda_{r}=\sqrt{\frac{2 G M}{r c^{2}}\left(1-\frac{G M}{2 r c^{2}}\right)}, \quad \lambda_{r}^{\prime}=\frac{\partial \lambda_{r}}{\partial r} .
\end{gathered}
$$

Applying (35) we obtain radial acceleration equation valid in the extremely strong static gravitational field

$$
\dot{\lambda}=0, \quad \rightarrow \quad \ddot{r}=-\frac{G M}{r^{2}}\left(1-\frac{G M}{r c^{2}}\right) .
$$

Proposition 4. The gravitational radial acceleration at the minimal gravitational radius is given by the relation:

$$
\begin{gathered}
\ddot{r}_{m}=\frac{4 c^{4}}{G M}=\rho_{r_{\max }}^{2} \frac{G}{M}=\frac{M_{p}}{L_{p}} \frac{4 c^{2}}{M}>0, \\
M=M_{p} \rightarrow \ddot{r}_{p m}=\frac{4 c^{2}}{L_{p}} .
\end{gathered}
$$

Here $\ddot{r}_{m}$ and $\ddot{r}_{p m}$ are the gravitational radial accelerations at the minimal gravitational radius and at the Planck's minimal radius $r_{p m}$, respectively, while $\rho_{r_{\max }}$ is the maximal radial density equal to the constant given by (32). As we can see from (37) the gravitational radial acceleration at the minimal gravitational radius is positive (repulsive) one and could be the source of the dark energy [18, 19].

Proof of the Proposition 4. In order to prove of the proposition 4, one can start with the equations for the minimal gravitational radius (18) and for maximal gravitational radial density (32):

$$
r_{m}=\frac{G M}{2 c^{2}}, \quad \rho_{r_{\max }}=\frac{M}{r_{m}}=\frac{2 c^{2}}{G}=\text { const } .
$$

Now, including of the minimal gravitational radius $r_{m}$ into the acceleration relation (36), one obtains the radial acceleration in gravitational field at the minimal radius:

$$
r=r_{m} \rightarrow \ddot{r}_{m}=-\frac{G M}{r_{m}^{2}}\left(1-\frac{G M}{r_{m} c^{2}}\right)=\frac{4 c^{4}}{G M}>0 .
$$

This equation can also be described as the function of the maximal gravitational radial density (32):

$$
r=r_{m} \rightarrow \ddot{r}_{m}=\frac{4 c^{4}}{G M}=\frac{2 c^{2}}{G} \frac{2 c^{2}}{G} \frac{G}{M}=\rho_{r_{\max }}^{2} \frac{G}{M}
$$

Another possibility is the description of the radial acceleration at the minimal gravitational radius as the function of the Planck's lent $L_{p}$ :

$$
\begin{aligned}
r & =r_{m} \rightarrow \ddot{r}_{m}=\frac{4 c^{4}}{G M}=\frac{2 c^{2}}{G} \frac{2 c^{2}}{M} \\
& =\frac{M_{p}}{r_{p m}} \frac{2 c^{2}}{M}=\frac{M_{p}}{L_{p}} \frac{4 c^{2}}{M} .
\end{aligned}
$$

It is easy to see that for the case where $M$ is equal to the Planck's mass $M_{p}$, the relation (41) is transformed into the radial acceleration $\ddot{r}_{p m}$ at the minimal gravitational radius of the Planck's mass $M_{p}$, as the only function of the Planck's lent $\mathrm{L}_{\mathrm{p}}$ : 


$$
\begin{aligned}
& r=r_{m} \rightarrow \ddot{r}_{m}=\frac{M_{p}}{L_{p}} \frac{4 c^{2}}{M}, \\
& M=M_{p} \rightarrow \ddot{r}_{p m}=\frac{4 c^{2}}{L_{p}} .
\end{aligned}
$$

Finally, the equations (39), (40) and (41) can be described by one relation:

$$
r=r_{m} \rightarrow \ddot{r}_{m}=\frac{4 c^{4}}{G M}=\rho_{r_{\max }}^{2} \frac{G}{M}=\frac{M_{p}}{L_{p}} \frac{4 c^{2}}{M}
$$

By the relations (42) and (43), the proof of the proposition 4 is finished.

Following the last relation in (43) one can see that the radial acceleration $\ddot{r}_{m}$ at the minimal gravitational radius is only function of the mass $M$. The other components are constants. In the case where $M<M_{p}$ the radial acceleration $\ddot{r}_{m}$ at the minimal gravitational radius is increasing to the maximal radial acceleration $\ddot{r}_{m_{\max }}$ at the minimal mass $M_{\text {min }}$. In the case where $M>M_{p}$ the radial acceleration $\ddot{r}_{m}$ at the minimal gravitational radius is decreasing to the minimal radial acceleration $\ddot{r}_{m_{\min }}$ at the maximal mass $M_{\text {max }}$. Finally, in the case where $M=M_{p}$ the radial acceleration $\ddot{r}_{m}$ at the minimal gravitational radius is equal to the radial acceleration $\ddot{r}_{p m}$ corresponding to the Planck's mass and given by the relation (42).

Now, we want to know the gravitational repulsive force at the minimal radius, $F_{m}$. Let this force accelerates mass $m=M$ to the acceleration $\ddot{r}_{m}$. Thus, for calculation of this force, we can employ relations in (43):

$$
\begin{aligned}
r & =r_{m} \rightarrow F_{m}=m \ddot{r}_{m}=M \ddot{r}_{m}=M \frac{4 c^{4}}{G M} \\
& =\frac{4 c^{4}}{G}=\rho_{r_{\max }}^{2} G=\frac{4 c^{2} M_{p}}{L_{p}}=\text { const } .
\end{aligned}
$$

As we can see from (44) the gravitational repulsive force at the minimal radius, $F_{m}$ (for $m=M$ ) is constant and the same for all mases $m_{i}=M_{i}$. Using standard units one can calculate this constant force:

$$
\begin{aligned}
r= & r_{m} \rightarrow F_{m}=m \ddot{r}_{m}=M \ddot{r}_{m}=\frac{4 c^{4}}{G} \\
& =48.410947907 \cdot 10^{43} \mathrm{~N}
\end{aligned}
$$

From the relation in (45) we can see that this is an enormous large force.

\section{CONCLUSION}

In this paper we considered the consequences of the existence of the minimal radius in gravitational field that has been predicted by the new Relativistic Alpha Field Theory
(RAFT). These consequences are in the connection with the belief that the Planck's length $L_{p}$, is the shortest meaningful length as the limiting distance below which any regular smaller distance in the spacetime does not exist. If RAF theory is correct then one can find out the following consequences of the existence of the minimal radius in a gravitational field. At the first each mass has its own minimal radius. The minimal radius of the Planck's mass is equal to half of the Planck's length. Thus, the physical significance of the Planck's length is that it is minimal (shortest) diameter of the Planck's mass. Further, the smallest minimal radius in a gravitational field belongs to the particle with the smallest mass. Since, the Planck's mass is not the smallest mass in the spacetime it follows that the Planck's length is not the shortest meaningful length in a spacetime. The radial density of each mass at its minimal gravitational radius is constant, equal to $2 c^{2} / G$, and the same and the maximal one for all pairs of masses and minimal gravitational radiuses. The gravitational radial acceleration at the minimal gravitational radius is positive (repulsive) one and could be the source of the dark energy.

\section{REFERENCES}

[1] Baez, J.: The Planck length. (1999). (http://math.ucr.edu/home/baez/planck/node2.html)

[2] CODATA: Constants, Planck length. US National Institute of Standard and Technology, June (2015). (http://physics.nist.gov/cgi-bin/cuu/Value?plkl)

[3] Carr, B. J., Giddings, S. B.: Quantum Black Holes, Scientific American 292, 48-55 (2005).

[4] Garay, L. J.: Quantum gravity and minimum length, International Journal of Modern Physics, A 10, 145 (1995). doi: $10.1142 / \mathrm{S} 0217751 \mathrm{X} 95000085$

[5] Burges, C., Quevedo, F.: The Grate Cosmic Roller-Coaster Ride. Scientific American (print). Scientific American, Inc. 55 (2007).

[6] Bowley, R., Eaves, L.: Planck Length. (2010). (http://www.sixtysymbols.com/videos/plancklength.htm)

[7] CODATA Value: Planck mass. US National Institute of Standard and Technology, June (2015). (http://physics.nist.gov/cgi-bin/cuu/Value?plkm)

[8] Bergmann, P. G.: The riddle of gravitation. Courier Corporation (1992). (https://books.google.com/books?id=WYxkrwMidp0C\&pg=PR10)

[9] Sivaram, C.: What is Special About the Planck Mass? arXiv:0707.0058v1 (2007).

[10] Einstein, A., Ann. Phys. 49, 769-822 (1916).

[11] Einstein, A.: The Meaning of Relativity. Princeton Univ. Press, Princeton (1955)

[12] Sean.C.: Spacetime and geometry: An introduction to general relativity. Amazon,com Books.htm, Hardcover (2003).

[13] Weinberg, S.: Gravitational and cosmology: Principles and application of the general relativity. Gebundene ausgabe,

[14] Hawking, S. W., Ellis, G. F. R.: The Large Scale Structure of Space-Time. Univ. Press, Cambridge (1973).

[15] Blau, M.: Lecture Notes on General Relativity. A. Einstein Center for Fundamental Physics, Univ. Bern, Bern (2014).

[16] Novakovic, B. M.: Relativistic alpha field theory - Part I: Determination of Field Parameters. International Journal of New Technology and Research (IJNTR) ISSN:2454-4116, 1, 5 (2015) 23-30. https://doi.org/10.31871/IJNTR.1.5.15

[17] Novakovic, B. M.: Relativistic alpha field theory-Part II: Does a Gravitational Field Could be Without Singularity? International Journal of New Technology and Research (IJNTR) (2015). https://doi.org/10.31871/IJNTR.1.5.16

[18] Novakovic, B. M.: Relativistic alpha field theory-Part III: Does Gravitational Force Becomes Positive if $\left(\mathrm{GM} / \mathrm{rc}^{2}\right)>1$ ? International Journal of New Technology and Research (IJNTR) (2015). https://doi.org/10.31871/IJNTR.1.5.17

[19] Novakovic, B. M. Is positive Gravitational Force Source of Dark 
Energy?, International Journal of New Technology and Research (IJNTR) ISSN:2454-4116, 1, 7 (2015) 6-13. https://doi.org/10.31871/IJNTR.1.7.5

[20] Novakovic, B. M.: Generalized Relativistic Hamiltonian in an Alpha Field. AIP Conference Proceedings 1303, 141 (2010). https://doi.org/10.1063/1.3527149

[21] Novakovic, B. M., Novakovic, D. B., Novakovic, A. B.: Cosmological Constant Problem Solution Valid for Both Planck's and Cosmological Scales. AIP Conference Proceedings 839, 144 (2006). https://doi.org/10.1063/1.2216624

[22] Perkins, D. H.: Introduction to High Energy Physics. Cambridge Univ. Press, Cambridge (2000).

[23] Sherman, D., et al. Nat. Phys. 11, 1-5 (2015).

[24] Meckel, M., et al. Nat. Phys. 10, 594 - 600 (2014).

[25] Novakovic, B. M.: Quantum Gravity in Relativistic Alpha Field Theory (QG in RAFT). International Journal of New Technology and Research (IJNTR) (2018). https://doi.org/10.31871/IJNTR.4.6.4

[26] Torres, R. Gen Relativ Gravit (2017) 49: 74. https://doi.org/10.1007/s10714-017-2236-5

[27] Garecki, J. Gen Relativ Gravit (1993) 25: 257. https://doi.org/10.1007/BF00756260

[28] Manfredi, G. Gen Relativ Gravit (2015) 47:1. https://doi.org/10.1007/s10714-014-1846-4

[29] Schiffer, M. Gen Relativ Gravit (1992) 24: 705. https://doi.org/10.1007/BF00760077

[30] Arbab, A.I. General Relativity and Gravitation (2004) 36: 2465. https://doi.org/10.1023/B:GERG.0000046833.08113.6b

[31] De Lorenzo, T., Pacilio, C., Rovelli, C. et al. Gen Relativ Gravit (2015) 47: 41.https://doi.org/10.1007/s10714-015-1882-8

[32] Li, H.L., Chen, SR. Gen Relativ Gravit (2017) 49: 128. https://doi.org/10.1007/s10714-017-2296-6

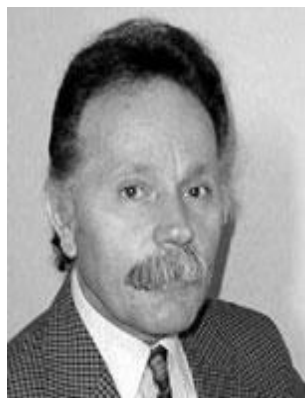

Branko Novakovic is a Professor emeritus at FSB - University of Zagreb, Croatia. Prof. Novakovic received his $\mathrm{PhD}$ from the University of Zagreb in 1978. His research of interest includes physics, control systems, robotics, neural networks, and fuzzy control. He is author of three books; Relativistic Alpha Field Theory (RAFT, e-book, 2016), Control Methods in Robotics, Flexible Manufacturing Systems and Processes (1990), Control Systems (1985) and the first co-author of a book Artificial Neural Networks (1998). He has published over 230 research papers in his research of interest. 